\title{
TEMPORAL ADJUSTMENT OF SHORT CALLS DURING VOCAL EXCHANGE IN JAPANESE MACAQUES
}

\author{
NORIKO KATSU $1^{* 1,2,3}$, KAZUNORI YAMADA $2^{2}$ KAZUO OKANOYA $3^{1}$, and \\ MASAYUKI NAKAMICHI $4^{2}$ \\ *Corresponding Author: katsuno0414@gmail.com \\ ${ }^{1}$ Graduate School of Arts and Sciences, University of Tokyo, Tokyo, Japan \\ ${ }^{2}$ Graduate School of Human Sciences, Osaka University, Osaka, Japan \\ ${ }^{3}$ Japan Society for the Promotion of Science, Tokyo, Japan
}

\section{Introduction}

In humans, turn-taking is a common feature during speech. Recent studies have revealed that nonhuman primates (Takahashi et al. 2013) and songbirds (Benichov et al. 2016) avoid overlap in vocal exchange and adjust response latency depending on the call of a partner. These studies also suggest the existence of a dynamic temporal adjustment in vocal exchange among nonhuman animals. Such vocal exchange occurs frequently among individuals with a strong social bond (Snowdon \& Cleaveland 1984).

Japanese macaques (Macaca fuscata) are known to reply to distance calls (coo calls) of group members after a certain period of latency (Sugiura 2007). Japanese macaques also use short-distance calls, such as grunts, girneys, and short low coos, in affiliative context. These calls are often emitted in a sequence, and are exchanged with a recipient (Katsu et al. 2017). Therefore, investigating vocal exchanges of these short-distance calls used during face-to-face interactions would help to understand social aspects of evolution of human conversation system. In the present study, we examined temporal adjustment in turn-taking of short-distance calls in Japanese macaques.

\section{Methods}

We conducted behavioral observations using a video camera on 15 adult female Japanese macaques in a free-ranging group in Kyoto, Japan. The group consisted 
of 126 members at the time of study. We recorded vocalizations including grunts, girneys, and coo calls of a focal subject facing another individual in the proximity of $5 \mathrm{~m}$ (a recipient), using a directional microphone and a digital audio recorder. We also recorded vocalizations from a recipient when the recipient replied within $5 \mathrm{~s}$. We used call bout as a unit for analyses, it is defined as a series of calls emitted in less than $5 \mathrm{~s}$ succession. We measured call intervals within a call bout, including inter- and intra-individual intervals.

\section{Results and Discussion}

We found few overlaps in calls during vocal exchanges in Japanese macaques. Distribution of intra-individual interval of spontaneous calls (i.e., interval between two consecutive calls without a reply from a recipient) showed periodicity of approximately $0.2 \mathrm{~s}$, which is close to the gap between turns in human conversation (0.25 s) (Stivers et al. 2009). There were substantial individual differences in the intervals of spontaneous calls, indicating that the regulation is necessary to avoid overlaps with calls from the recipient.

We then examined if the Japanese macaques adjusted call timing according to the response latency of a partner. We calculated interval between (a) a spontaneous call of a subject and a reply from a recipient, (b) the spontaneous call and a call of the subject after the reply from the recipient, and (c) two spontaneous calls of the subject without replies (Takahashi et al. 2013). The differences between (b) and (c) indicated an amount of temporal adjustment in vocal exchange when compared with that of spontaneous calls, and (a) indicated response latency from a recipient. The analyses revealed that (b)-(c) the amount of temporal adjustment was significantly affected by (a), that is, response latency from a recipient. This finding supports that they adjusted call timing according to the recipient. Japanese macaques are non-vocal learners, however, the present study showed that they have some temporal flexibility in vocalization. A previous study showed that affiliative interactions, such as grooming were more likely to occur when there was vocal exchange (Katsu et al. 2016). Such vocal timing flexibility might play an important role in social coordination in nonhuman primates, and needs further investigation to understand evolution of form and function of turn-taking in human speech.

\section{Acknowledgments}

We are grateful for the support provided by Mr. S. Asaba and the staff of the Iwatayama Monkey Park, Japan. Financial support was provided by JSPS KAKENHI Grant \#17J10994 to NK and MEXT grant on Innovative Areas \#4903, $17 \mathrm{H} 06380$ to $\mathrm{KO}$. 


\section{References}

Benichov, J., Benezra, S., Vallentin, D., Globerson, E., Long, M. \& Tchernichovski, O. (2016). The forebrain song system mediates predictive call timing in female and male zebra finches. Current Biology, 26, 309-318.

Katsu, N., Yamada, K., \& Nakamichi, M. (2016). Function of grunts, girneys and coo calls of Japanese macaques (Macaca fuscata) in relation to call usage, age and dominance relationships. Behaviour, 153, 125-142.

Katsu, N., Yamada, K., \& Nakamichi, M. (2017). Influence of social interactions with nonmother females on the development of call usage in Japanese macaques. Animal Behaviour, 123, 267-276.

Snowdon, C.T., \& Cleveland, J. (1984). "Conversations" among pygmy marmosets. American Journal of Primatology, 7, 15-20.

Stivers, T., Enfield, N.J., Brown, P., Englert, C., Hayashi, M., Heinemann, T., Hoymann, G., Rossano, F., de Ruiter, J., Yoon, K-E., \& Levinson, S.C. (2009). Universals and cultural variation in turn-taking in conversation. Proceedings of the National Academy of Sciences of the United States of America, 106, 10587-10592.

Sugiura, H. (2007). Adjustment of temporal call usage during vocal exchange of coo calls in Japanese macaques. Ethology, 113, 528-533.

Takahashi, D.Y., Narayanan, D.Z., \& Ghazanfar, A.A. (2013). Coupled oscillator dynamics of vocal turn-taking in monkeys. Current Biology, 23, 2162-2168. 